\title{
Shedding Light on Lipase Stability in Natural Deep Eutectic Solvents
}

\author{
A. A. Elgharbawy, ${ }^{a}$ A. Hayyan, ${ }^{b, c, *}$ M. Hayyan, ${ }^{c, d, *}$ S. N. Rashid, \\ M. R. M. Nor, ${ }^{\mathrm{e}}$ M. Y. Zulkifli, ${ }^{\mathrm{e}}$ Y. Alias, ${ }^{\mathrm{c}, \mathrm{f}}$ and M. E. S. Mirghania ${ }^{\mathrm{a}, \mathrm{g}}$ \\ ${ }^{a}$ International Institute for Halal Research and Training (INHART), \\ International Islamic University Malaysia, Kuala Lumpur 50728, Malaysia \\ ${ }^{b}$ Nanotechnology \& Catalysis Research Centre (NANOCAT), \\ University of Malaya, Kuala Lumpur 50603, Malaysia \\ 'University of Malaya Centre for Ionic Liquids (UMCiL), \\ University of Malaya, Kuala Lumpur 50603, Malaysia \\ ${ }^{\mathrm{d} D e p a r t m e n t}$ of Chemical Engineering, Faculty of Engineering, \\ Sohar University, P.O. Box 44, Sohar P.C. 311, Sultanate of Oman \\ ${ }^{\mathrm{e} H a l a l}$ Research Group, Academy of Islamic Studies, \\ University of Malaya, 50603, Kuala Lumpur, Malaysia \\ ${ }^{\mathrm{f}}$ Department of Chemistry, Faculty of Science, \\ University of Malaya, 50603 Kuala Lumpur, Malaysia \\ gDepartment of Biotechnology Engineering, \\ Kulliyyah of Engineering, International Islamic \\ University Malaysia, Kuala Lumpur 50728, Malaysia
}

doi: 10.15255/CABEQ.2018.1335

Original scientific paper

Received: March 22, 2018

Accepted: September 25, 2018

\begin{abstract}
This study presents the potential role of natural deep eutectic solvents (NADESs) in a lipase-catalyzed hydrolysis reaction as both a co-solvent in an aqueous solution and as a main solvent. Ammonium salts such as choline chloride $(\mathrm{ChCl})$ were paired with different hydrogen bond donors such as glycerol and malonic acid and sugars (glucose, fructose and sucrose). The hydrolysis of $p$-nitrophenyl palmitate by six different lipases: lipase from porcine pancreas (PR), lipase from Candida rugosa (CR), Amano lipase PS, from Burkholderia cepacia (AM), lipase from Rhizopus niveus (RN), lipase acrylic resin from Candida antartica (ARC), lipase B Candida antartica immobilized on Immobead 150, recombinant from Aspergillus oryzae (CALB), were tested in five NADESs. The results showed that NADES3 prepared from $\mathrm{ChCl} /$ sucrose was the most promising solvent as it enhanced the activities of both CALB and lipase from porcine pancreas to $355 \%$ and $345 \%$. The kinetics investigation confirmed the higher catalytic efficiency $\left(k_{\text {cat }} / K_{\mathrm{m}}\right)$ of lipases in the $40 \%$ of (NADES3) and compared with the aqueous form. The trend achieved by NADES may be a promising approach for applications and further perspectives as genuinely green industrial solvents.
\end{abstract}

Keyword:

biotransformation, biotechnology, ionic liquids, choline chloride, $p$-nitrophenyl palmitate, lipase B Candida antartica

\section{Introduction}

Over the last few years, the use of deep eutectic solvents (DESs) as a green alternative to conventional solvents has been dramatically expanded ${ }^{1}$. Recently, Dai and collaborators have introduced various stable natural deep eutectic solvents (NADESs) based on natural compounds ${ }^{2}$. NADESs present a new generation of liquid salts, and they

*Corresponding authors: adeeb.hayyan@yahoo.com;

maan_hayyan@yahoo.com are generally based on mixtures of relatively cheap and readily available components, such as non-toxic quaternary ammonium salts (e.g., choline chloride) with naturally derived uncharged hydrogen-bond donors such as vitamins, amines, sugars, alcohols, and carboxylic acids. The composition of NADESs results in low production cost and high safety due to the low volatility and absence of toxicity for both workers and end users ${ }^{3}$. NADESs have unique physicochemical properties, with the possibility for their design for particular purposes, and thus, along with other environmental benefits of using NA- 
DESs as solvents, such as their low vapor pressures and non-flammability, NADESs represent excellent candidates for green extractions ${ }^{4}$. NADESs are more biodegradable, biocompatible, and safe compared with normal DESs. Those features render them suitable as solvents for biocatalytic reactions, practically in the pharmaceutical, nutraceutical, and cosmetic industries where product biocompatibility is a major issue ${ }^{4}$.

Since their emergence, NADESs have attracted attention as potential solvents in various industrial fields, including the extraction of biologically active compounds from plant materials ${ }^{5}$, solubilization of cellulose, gluten, starch and $\mathrm{DNA}^{6}$, organic reaction media, and in electrochemical applications due to their conductivity ${ }^{7}$.

NADESs based on glycerol may be practical substitutes for biodiesel production in the biocatalytic system. For that purpose, lipases are widely applied in the enzymatic process. Choline chloride-based DES was used by Zhao et al. ${ }^{8}$ In this study, they demonstrated that CALB could maintain high activity and could be introduced in the enzymatic transesterification of triglycerides with ethanol, to obtain high yields.

Indeed, several reports have demonstrated that hydrolases or proteases preserved high activity in choline or ethyl ammonium chloride-based DESs that are paired with hydrogen-bond donors such as alcohols, acids or amides 9 . DESs have also been used efficiently in lipase-catalyzed processes, resulting in high enzyme stability. $\mathrm{ChCl} /$ glycerol (1:2) and ChOAc/glycerol (1:1.5) were biocompatible with immobilized Candida antartica lipase B $(\mathrm{CALB})^{8,10}$. Furthermore, choline chloride/glycerol (1:2 molar ratio) was evaluated as a co-solvent in Novozym 435-catalyzed transesterification of soybean oil with methanol to achieve up to $88 \%$ triglyceride conversions in $24 \mathrm{~h}^{8}$. Taken together, the biocompatibility as well as the sustainability of NADESs, render them as an excellent substitute for the aqueous media for biomolecules ${ }^{7}$.

In this context, it seems very promising to develop a lipase-compatible NADES from inexpensive and biodegradable salts. In one of the previous studies, lipase B of Candida antarctica (CALB) proved to be the best option to carry out biocatalysis reaction in $\mathrm{DESs}^{11}$. However, due to insufficient reports with other lipases, it is interesting to investigate the potential of NADESs as media for biotransformation using various lipases. We investigated the activity and stability of six lipases; lipase from porcine pancreas (PR), lipase from Candida rugosa (CR), Amano lipase (AM), lipase from Rhizopus niveus (RN), lipase acrylic resin from Candida antartica (ARC), Lipase B Candida antartica immobilized on Immobead, recombinant from $\mathrm{As}$ - pergillus oryzae (CALB), in five choline chloride-based NADESs paired with sugars, glycerol, and malonic acid. This study focuses on the activation and stability after incubation of the selected lipases in NADESs.

\section{Materials and methods}

\section{Biochemical materials}

The primary substrate, $p$-nitrophenyl palmitate, $p$-nitrophenol, isopropanol, sodium deoxycholate (purity $\geq 97 \%$ ), choline chloride (purity $\geq 98 \%$ ), was purchased from Sigma-Aldrich (US). Other chemicals were purchased from MERCK (Germany) and HmbG Chemicals (Hamburg) and were of analytical grade. Lipases; lipase from porcine pancreas (PR) (100-500 units/mg protein), lipase from Candida rugosa (CR) ( $\geq 700$ unit $\mathrm{mg}^{-1}$ solid), Amano lipase PS, from Burkholderia cepacia (AM) $\left(\geq 30,000 \mathrm{U} \mathrm{g}^{-1}\right)$, lipase from Rhizopus niveus (RN) $\left(\geq 1.5 \mathrm{U} \mathrm{mg}^{-1}\right)$, lipase acrylic resin from Candida antartica (ARC) $\left(\geq 5,000 \mathrm{U} \mathrm{g}^{-1}\right.$, recombinant, expressed in Aspergillus niger), Lipase B Candida antartica immobilized on Immobead 150, recombinant from Aspergillus oryzae (CALB) $\left(\geq 1800 \mathrm{U} \mathrm{g}^{-1}\right)$, were all obtained from Sigma-Aldrich.

\section{Preparation of NADESs}

Choline chloride $(\mathrm{ChCl})$ and an HBD (structures are presented in Table 1), were mixed at a specific molar ratio, as indicated in Table 2, in autoclaved, dry and dark flasks. $\mathrm{ChCl}$ was dried in a vacuum dryer at $80{ }^{\circ} \mathrm{C}$ for six hours prior to use because of its hygroscopic nature. The flasks were then heated and stirred at the speed of $350 \mathrm{rpm}$ and temperature of $80{ }^{\circ} \mathrm{C}$ for two hours in an incubator shaker until the NADESs became homogeneous and stable with no visible precipitate. The water content of all NADESs ranged from $0.43-1.23 \%$ as determined by Karl-Fischer titration. All the synthesized NADESs were kept in controlled moisture environment for further use. Prior to use, the NADESs were dried at $80{ }^{\circ} \mathrm{C}$ to remove any moisture interference. Addition of distilled water was included in the synthesis of aqueous NADESs based on a molar ratio with HBA or salt. The water was poured before mixing both the HBD and $\mathrm{HBA}^{12}$.

\section{Lipase activity assay}

Lipase enzyme activity in aqueous solutions was determined using calorimetric assay. Hydrolysis of $p$ NPP to $p$-nitrophenol was selected as a standard reaction to determine lipase activity. The enzyme substrate was prepared accordingly $(7 \mathrm{mg}$ of $p$ NPP was dissolved in $4 \mathrm{~mL}$ of isopropanol) and 
Table 1 -Structures, names, and abbreviations of the individual components of the tested NADESs

\begin{tabular}{c|c}
\hline Ammonium Salt & Name \\
\hline \\
\hline
\end{tabular}<smiles>O=C(CO)[C@H](O)[C@H](O)[C@H](O)CO</smiles>

Fructose<smiles>O=C(O)CC(=O)O</smiles>

Malonic acid

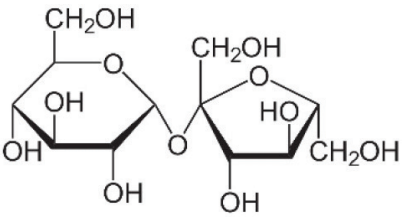

Sucrose<smiles>O=CC(O)[C@H](O)[C@H](O)[C@H](O)CO</smiles>

Glucose<smiles>OCC(O)CO</smiles>

Glycerol

Table 2 -Compositions and molar ratios of the tested NADESS

\begin{tabular}{l|l|l|c|c}
\hline \multicolumn{1}{c|}{ Type } & $\begin{array}{c}\text { NADES } \\
\text { code }\end{array}$ & DES composition & $\begin{array}{c}\text { Molar } \\
\text { ratio }\end{array}$ & pH \\
\hline \multirow{5}{*}{$\begin{array}{l}\text { NADES1a } \\
\text { (Aqueous) }\end{array}$} & NADCl: fructose: water & $5: 2: 5$ & 1.96 \\
& NADES2a & ChCl: malonic acid & $1: 1$ & 0.00 \\
& NADES4a & ChCl: glycerol: water & $1: 2: 1$ & 1.98 \\
& NADES5a & ChCl: glucose: water & $5: 2: 5$ & 2.32 \\
\hline & NADES1 & $\mathrm{ChCl}$ : fructose & $5: 2$ & 2.08 \\
& NADES2 & $\mathrm{ChCl}$ : malonic acid & $1: 1$ & 0.00 \\
$\begin{array}{l}\text { Binary } \\
\text { (Non- } \\
\text { aqueous) }\end{array}$ & NADES3 & $\mathrm{ChCl}$ : sucrose & $4: 1$ & 3.40 \\
& NADES4 & $\mathrm{ChCl}$ : glycerol & $1: 2$ & 2.46 \\
& NADES5 & $\mathrm{ChCl}$ : glucose & $5: 2$ & 2.18 \\
\hline
\end{tabular}

mixed with $65 \mathrm{~mL}$ of the sodium phosphate buffer ( $\mathrm{pH} 8.0 \pm 0.2$ ). To the buffer solution, $0.14 \mathrm{~g}$ of sodium deoxycholate, $70 \mathrm{mg}$ of Arabic gum, and $0.35 \mathrm{~mL}$ of Triton X-100 (to remove turbidity) were added. The final mixture was gently mixed and used freshly for every assay. The reaction was started by the addition of $300 \mu \mathrm{L}$ lipase or lipase/NADES mixture, then, $700 \mu \mathrm{L}$ of freshly prepared substrate solution was added. The tubes were incubated in a water bath and warmed at $40{ }^{\circ} \mathrm{C}$ for $15 \mathrm{~min}$ before it was stopped by adding $300 \mu \mathrm{L}$ of (1:1) ethanol/ acetone solution ${ }^{13}$. The absorbance of $p$-nitrophenol $(p \mathrm{NP})$ was monitored using a Multiskan ${ }^{\mathrm{TM}} \mathrm{GO}$ microplate spectrophotometer at $410 \mathrm{~nm}$. Each assay was measured in triplicate for each DES, and the results are presented as the mean of the obtained data \pm standard deviation (SD). As the molar extinction coefficient can vary due to the presence of NADES and different $\mathrm{pH}$ values, individual standard curves were generated for each NADES/buffer solution. The absorbance of $p \mathrm{NP}$ was measured at $410 \mathrm{~nm}$, and then the molar extension coefficient was calculated accordingly. Enzyme activity is expressed as unit $\mathrm{mg}^{-1}$. One unit was defined as the amount of enzyme that liberated one mole of $p \mathrm{NP}$ per minute. The absorbance of the NADES without enzyme was subtracted for value correction.

\section{Activity of lipases in NADESs}

Lipases were suspended in a phosphate buffer and various NADESs with fixed enzyme concentrations [1 mg lipase/mL (solvent/buffer)] for an hour. All the solutions were incubated at $40{ }^{\circ} \mathrm{C}, 300 \mathrm{rpm}$ in a thermomixer (Eppendorf Comfort). The lipase assay was performed as explained previously.

\section{Factors influencing lipase activity}

The effects of $\mathrm{pH}$, temperature, and water content were all investigated by incubating each lipase/ NADES mixture for an hour in varied conditions (temperature, $\mathrm{pH}$, water content) by taking the control reading when the enzyme was at optimum conditions (optimum temperature, $\mathrm{pH}$ ). Lipases investigated in this study were reported to be at maximum activity at $\mathrm{pH} 7.0-8.0$ in general, as listed in the product specification (Sigma Aldrich). The solution after suspension was used for the assay and related to the initial activity to calculate the relative activity. Buffers used in this study were citrate-phosphate buffer $(\mathrm{pH}$ 5.0-6.0), sodium phosphate buffer $(\mathrm{pH}$ 7.0-8.0) and glycine-sodium hydroxide buffer $(\mathrm{pH}$ 9.0-10.0). NADES has low $\mathrm{pH}$ values, therefore, the $\mathrm{pH}$ values of the reaction media were adjusted using 1.0 M NaOH to achieve the required value.

Water content effect was compared for both aqueous and non-aqueous NADESs in various con- 
centrations (\% NADES/water). After incubation for one hour, the lipase assay was performed as previously described.

For the thermal stability and $\mathrm{pH}$ stability, lipases that were pre-incubated at different temperatures (in phosphate buffer and NADES) and several $\mathrm{pH}$ values (5.0-10.0) for 12 hours were assessed by determining the residual and relative activities. The residual activity is defined as the percentage (\%) of lipase activity after incubation relative to the initial activity of lipase without incubation, while relative activity is the percentage (\%) of lipase activity in reference to the lipase incubated at the same conditions in buffer solution ${ }^{14}$.

The stability during storage was assessed after 72 hours of pre-incubation at $4.0 \pm 2.0{ }^{\circ} \mathrm{C}$. The lipases were suspended in phosphate buffer, NADES3 and NADES3a, samples were subjected to lipase assay after incubation and the results were recorded as relative activity (\%).

\section{Kinetic parameters of lipases in NADESs}

Kinetic parameters were calculated at substrate concentrations ranging from 0.3 to $4.0 \mathrm{mM}$. Kinetic parameter determination was performed at the optimal temperature and $\mathrm{pH}$ for each lipase. The kinetic curve was developed based on the experimentally obtained data. Kinetic parameters, Michaelis-Menten constant $\left(K_{\mathrm{m}}\right)$, and maximum velocity $\left(V_{\max }\right)$, were calculated by plotting the kinetic data according to the Lineweaver-Burk methodology. As the total amount of enzyme used is known, both the turnover number $\left(k_{\text {cat }}\right)$ and the catalytic efficiency $\left(k_{\text {cat }} / K_{\mathrm{m}}\right)$ were determined.

\section{Results}

\section{Screening of different NADESs for enzyme activity}

Fig. 1 shows that the activity of lipases fluctuates in the different types of NADESs. However, it can be observed that sugar-based NADESs were more stabilizing than other NADESs used in this study. One-Way ANOVA Tukey's test with a confidence level of $96 \%$ was performed to obtain the possible grouping from these data. The analysis revealed that all results were significantly different from each other as they fall into different groups (letters). The similarities were noticed in porcine and amano lipase, as they both showed a stabilizing effect closer to the buffer (100\%). However, lipases showed a different attitude in NADES2a, NADES3, and NADES5a. From Fig. 1 and the analysis of data, generally, NADES2a showed inhibitory effect, while it was observed that NADES1a stabilized most of the lipases. On the other hand, NADES3a

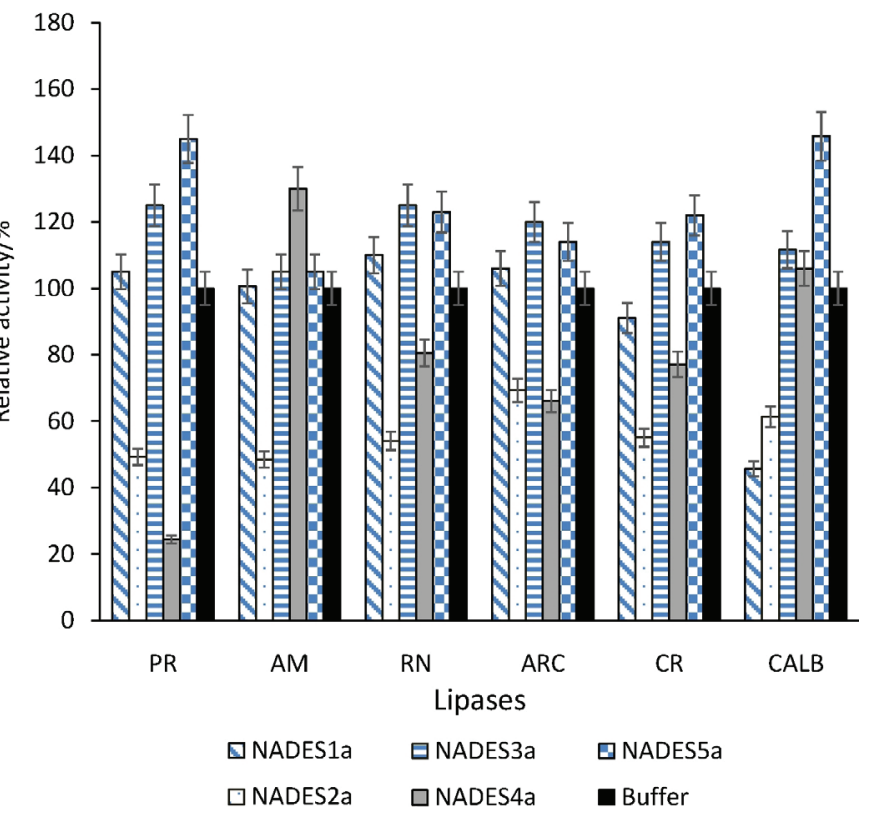

Fig. 1 - Screening of different lipases with several NADESs. Phosphate buffer ( $p H$ 7.0) was used as a reference for each lipase's control as a comparison. Lipase from porcine pancreas $(P R)$, lipase from Candida rugosa (CR), Amano lipase PS, from Burkholderia cepacia (AM), lipase from Rhizopus niveus (RN), lipase acrylic resin from Candida antartica (ARC) (recombinant, expressed in Aspergillus niger), Lipase B Candida antartica immobilized on Immobead 150, recombinant from Aspergillus oryzae (CALB).

and NADESa5 activated most of the lipases. NADES4a had an inhibitory effect on most of the lipases except for CALB and amano lipase (AM). This could be attributed to water content, as shown by Zhao et al. ${ }^{15}$ that activity and selectivity of proteases in glycerol-based DESs are dependent on the water content, whereby a small amount of water of about $5 \%$ can increase the enzymatic activity. CALB is less likely to be highly affected due to the immobilization and AM lipase and showed high stability in most of the tested NADESs. The inhibitory effect of NADES4a may be due to the change in polarity associated with increasing the water content $(>4 \%)$ which may affect the selectivity of the lipase towards the substrate, $p$ NPP, in this case.

As the results in the first round were promising, the second round of screening was executed because of the exciting trends observed with the sugar-based NADESs. Overall, NADES1a significantly enhanced the activities of porcine (PR), Rhizopus (RN) and resin (ARC) lipases, but it showed inhibitory effect on other lipases.

The NADES3a had a more enhancing effect compared with NADES5a although both had activated all lipases in the screening. However, from the observed trend in Fig. 2, NADES3a is more capable of activation than NADES5a. 


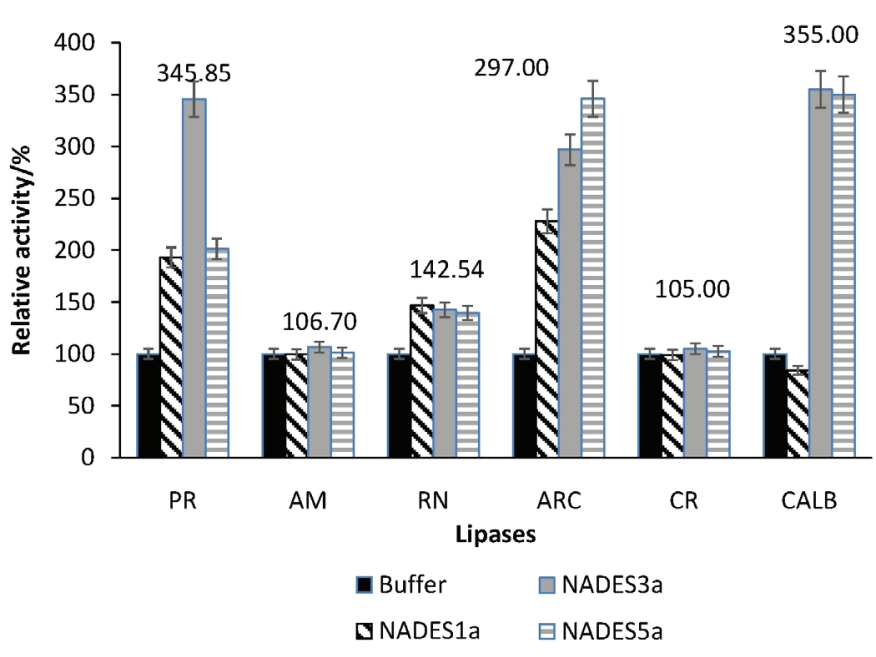

Fig. 2 - Screening of different lipases with sugar-based NADESs against buffer as a control reference. Lipase from porcine pancreas (PR), lipase from Candida rugosa (CR), Amano lipase PS, from Burkholderia cepacia (AM), lipase from Rhizopus niveus $(R N)$, lipase acrylic resin from Candida antartica (ARC) (recombinant, expressed in Aspergillus niger), Lipase B Candida antartica immobilized on Immobead 150, recombinant from Aspergillus oryzae (CALB).

Compared to untreated enzymes, lipases pretreated with NADES3a were activated; as observed from Fig. 2, CALB, PR, ARC, and RN are enhanced up to $355 \%, 345 \%, 297 \%$, and $142.5 \%$, respectively. In DES5, a similar trend was observed with lower activation effect as the highest was detected with CALB and ARC, $350 \%$ and $346 \%$ respectively.

From Tukey's test, it can also be concluded that the stimulatory effect of both NADES3a and NADES5a can be categorized into one group since they are close to each other (sharing the same letter). However, since the highest activity was obtained when those lipases were incubated with NADES3a, and NADES1a showed some level of inhibition towards CALB in contrast with others, NADES3a was selected for further investigation. CALB has been detailed in many studies because its protein exhibited an excellent substrate specificity, and was profoundly active towards an assortment of amides, esters, and thiols. Due to the active site made up of typical catalytic triad (Ser-Asp-His) near two flexible a-helixes, CALB houses many different substrates $^{16}$ and is well documented.

It was demonstrated that high activity of $\mathrm{Can}$ dida antarctica lipase A and B (CALA and CALB) was detected in NADESs composed of choline chloride or ethyl ammonium chloride paired with H-bond donors such as acetamide, ethylene glycol, glycerol, urea, and malonic acid ${ }^{11}$. They observed that CALB was 20-35-fold more stable in the DES $\mathrm{ChCl} / \mathrm{U}$ than in an aqueous solution of the DES components which denatured or deactivate the enzyme. The strong hydrogen-bond network explains this surprising stability within the DES, which lowers the diffusion of the constituents to the protein core. The DES forms hydrogen bonds with the surface residues of the enzyme, which, instead of denaturation, may stabilize the tertiary structure of the enzyme ${ }^{17}$.

In general, a wide range of variation in lipases activity was observed with different NADESs used for pretreatment (Fig. 1). A reduction in some lipases activity was observed in the case of NADES2a and NADES4a containing malonic acid and glycerol, which can be seen in Fig. 1. However, NADES2a was the most inhibitory agent among all DESs tested.

\section{Effect of $\mathrm{pH}$}

The optimum $\mathrm{pH}$ values in the presence of different types of NADESs as co-solvents in aqueous solution was studied as illustrated in Fig. 3.

The current results in Fig. 3 agreed with the reported data as lipases were active at around the optimum values. However, the activity of lipases slightly increased, and the enzyme performed better at different $\mathrm{pH}$ value compared with that of buffer solutions. For instance, ARC lipase was more active at $\mathrm{pH}$ 7.0. However, its activity was stimulated at both $\mathrm{pH} 7.0$ and 8.0 after incubation with NADES3a. PR lipase's optimum $\mathrm{pH}$ did not change, but its activity improved.

After adding NADES3a as a co-solvent to the reaction mixture, a significant increment in enzyme activity (2-3 fold) was observed in general. It was also noticed that lipases/NADES3a were more stable at $\mathrm{pH} 9.0$ in contrast with free lipases. Moreover, both CR and CALB exhibited a significant improvement and a shift in $\mathrm{pH}$ values for their maximum activities. Nevertheless, $\mathrm{pH} 10.0$ was not a suitable medium for lipase activity neither in the buffer solutions nor in the NADES3a. The stability was further observed for 12 hours where lipases were active in the $\mathrm{pH}$ range $6.0-8.0$, with no loss of activity at 7.0-8.0. CALB recorded high activity in the range of 7.0-9.0. The activity and stability shift could be attributed to the nature of NADES and the strong polarity due to the multiple hydroxy groups ${ }^{18}$ in the sugar-based NADES.

\section{Effect of temperature}

Further investigation was executed to study the effect of reaction temperature on enzyme activity. The results obtained showed a typical enzyme activity trend with a variation of optimal reaction temperatures for each lipase in the range of 30 to $80^{\circ} \mathrm{C}$ (as shown in Fig. 4a). When the reaction tempera- 


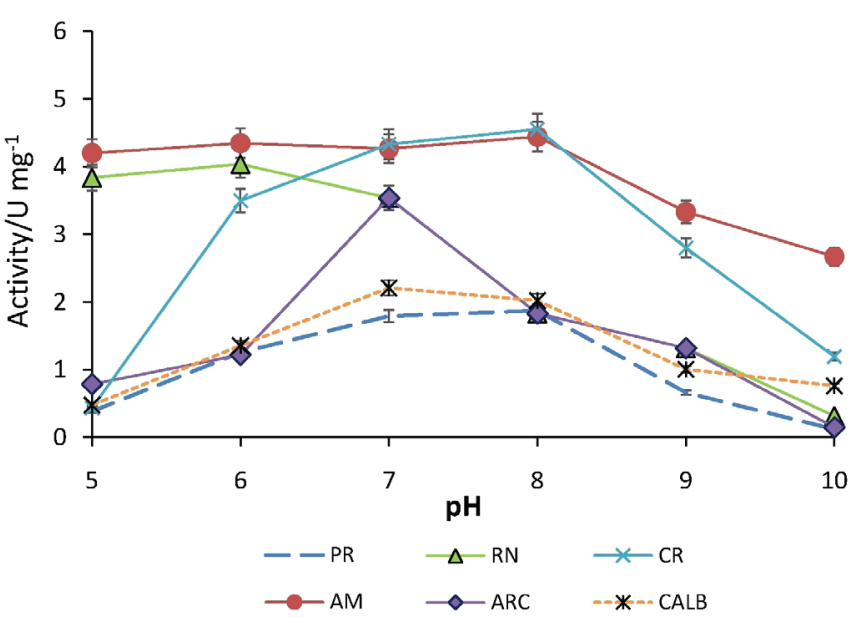

Fig. 3 a - Activity of lipases at different $p H$ values (incubation in buffer solution)

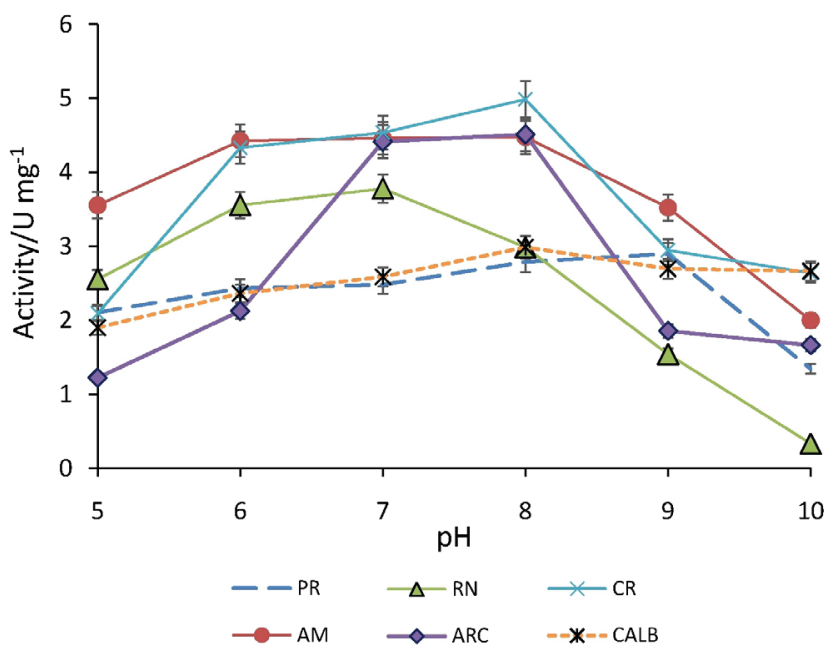

Fig. $3 \mathrm{~b}-$ Activity of lipases at different $\mathrm{pH}$ values in the presence of NADES3

ture increased, enzyme activity gradually increased up to certain levels (around $50{ }^{\circ} \mathrm{C}$ ). However, the reaction rate decreased clearly at elevated temperatures due to denaturation of the protein. The same pattern was also reported for Burkholderia cepacia lipase (BCL) in $\mathrm{ChCl}$ : $\mathrm{EG}^{19}$.

Comparison of the initial activity among the tested temperatures for the six lipases revealed that PR lipase achieved the maximum activity. Furthermore, all lipases possessed their optimal activities at $40{ }^{\circ} \mathrm{C}$, except for PR lipase, which was at $50{ }^{\circ} \mathrm{C}$. After the pretreatment in NADES3a for an hour, the lipases showed a different trend (Fig. 4b) in contrast with their control samples.

In NADES3a (aqueous media), lipases including $\mathrm{RN}, \mathrm{AM}, \mathrm{CR}$, and $\mathrm{ARC}$, possessed a higher activity than in the buffer solution at $50{ }^{\circ} \mathrm{C}$. Both CALB and PR lipases exhibited enhancement of activity at $60^{\circ} \mathrm{C}$. Significant activation was achieved

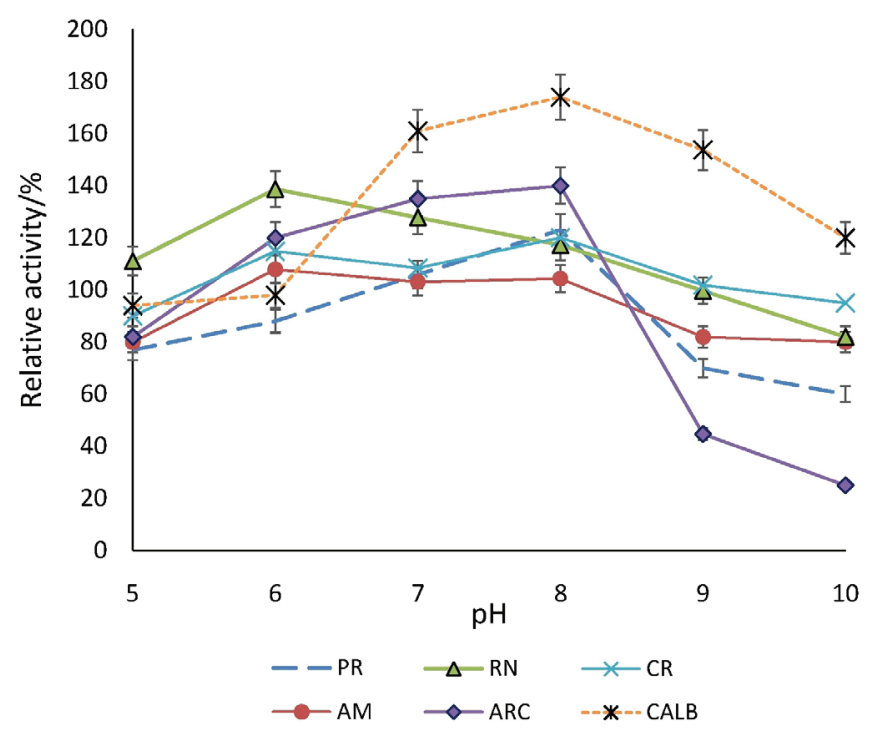

Fig. 3c - Stability of lipases at different $p H$ values in the presence of NADES3 after 12 hours. $40^{\circ} \mathrm{C}$. Lipase from porcine pancreas (PR), lipase from Candida rugosa (CR), Amano lipase PS, from Burkholderia cepacia (AM), lipase from Rhizopus niveus (RN), lipase acrylic resin from Candida antartica (ARC) (recombinant, expressed in Aspergillus niger), Lipase $B$ Candida antartica immobilized on Immobead 150, recombinant from Aspergillus oryzae (CALB).

by incubating CALB and RN in NADES3a as it increased up to $243 \%$ and $236 \%$ at $60{ }^{\circ} \mathrm{C}$ and $50{ }^{\circ} \mathrm{C}$, respectively.

Thermal stability of lipases was further investigated (Fig. 4c and 4d) for 12 hours in the range of $30-60{ }^{\circ} \mathrm{C}$. While more than $50 \%$ of the activity was lost above $50{ }^{\circ} \mathrm{C}$ in buffer, lipases shifted the optimal temperature at $50^{\circ} \mathrm{C}$ and CALB was active at $60{ }^{\circ} \mathrm{C}$ with no loss detected. Above $45{ }^{\circ} \mathrm{C}$, the residual activity of lipase in $10 \%[\mathrm{Ch}] \mathrm{Cl} /$ glycerol after $1 \mathrm{~h}$ of incubation was about 2-fold higher than that of lipase (Candida rugosa) in buffer without additive, as reported by Kim et al. ${ }^{14}$

A conceivable explanation for the expanded thermal stability of the enzyme in DES is that DESs kept protein from irreversible aggregation at high temperatures and thus increased the stability ${ }^{20}$. The hydrogen bonding in DESs helps to preserve the 3D structure of the enzyme at high temperature. Moreover, it might also play an important role in maintaining the integrity of the native conformation of the enzyme and preventing the collapse of the enzyme framework at a higher temperature by absorbing the heat from the reaction medium ${ }^{21}$.

If the concept was applied, lipases inactivation occurred at $70{ }^{\circ} \mathrm{C}$, but with the addition of NADES3a (sucrose-based), the activity in this study was retained at a high temperature of $80{ }^{\circ} \mathrm{C}$ with almost full recovery of activities of the six lipases used. This can be explained by the fact that the elevated 


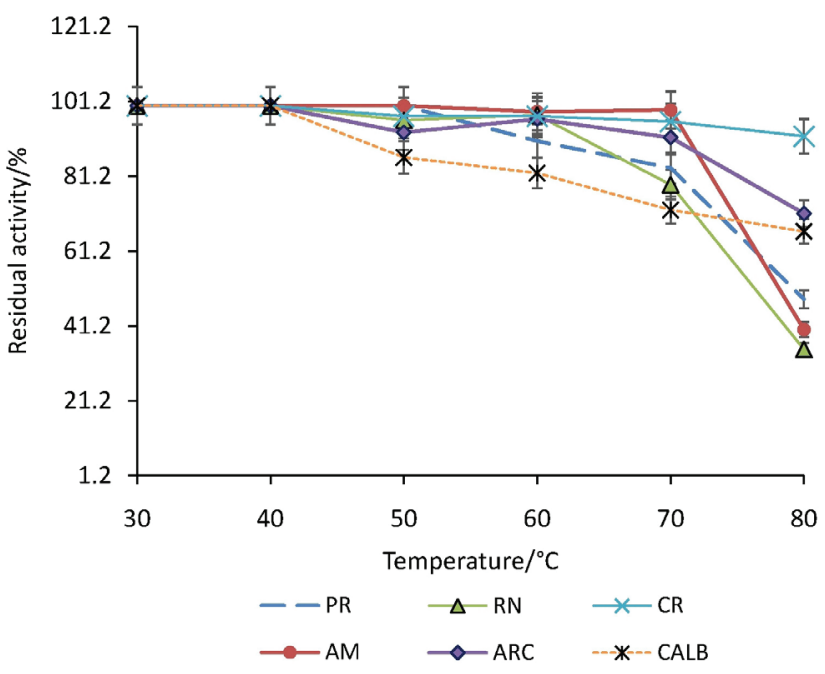

Fig. 4 a - Activity of lipases at different temperatures (incubation in buffer solution)

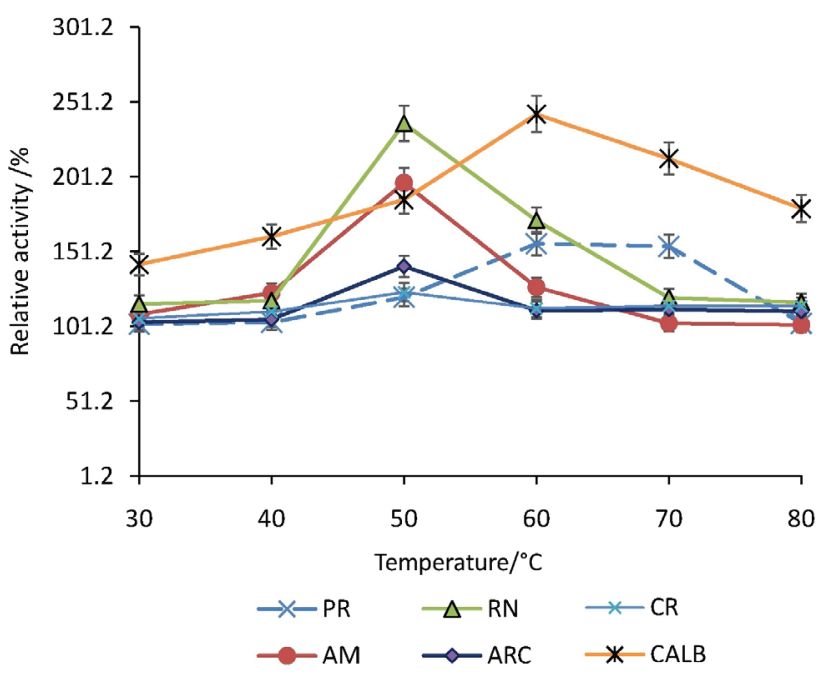

Fig. 4b-Relative activity of lipases at different temperatures in the presence of $40 \%$ NADES3 compared to the initial activity after incubation for one hour

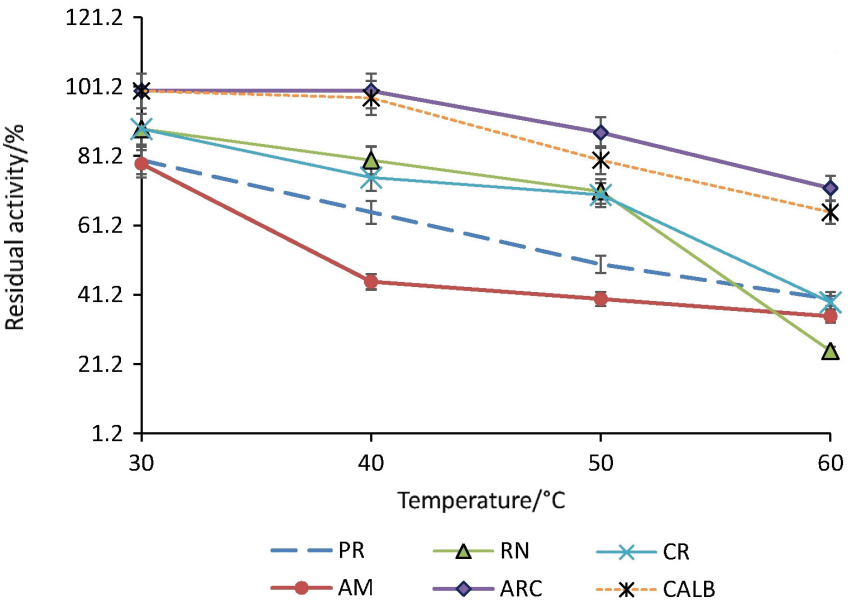

Fig. 4c - Stability of lipases at different temperatures in phosphate buffer compared to the initial activity after incubation for 12 hours

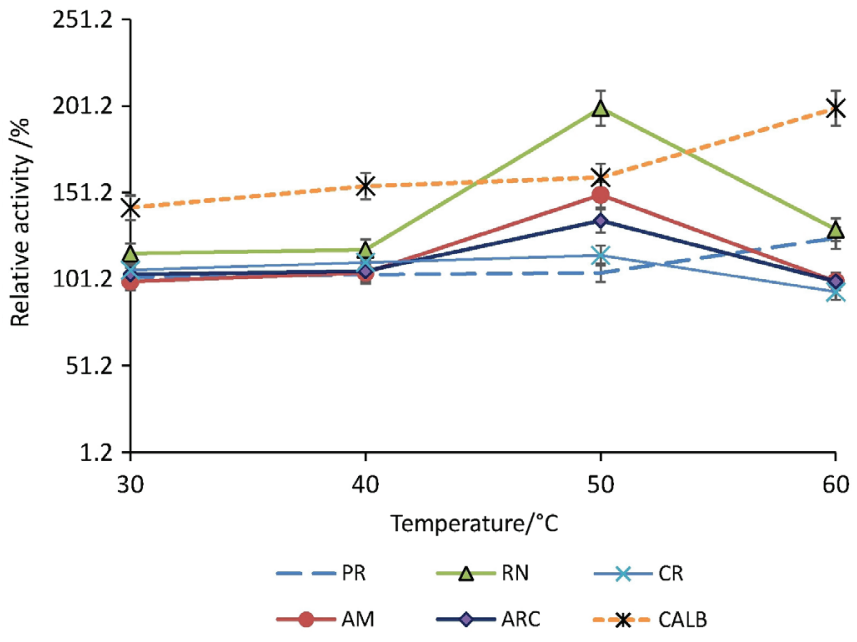

Fig. 4d - Stability of lipases at the optimal range of temperatures in the presence of $40 \%$ NADES3 compared to the initial activity after incubation for 12 hours. Lipase from porcine pancreas (PR), lipase from Candida rugosa (CR), Amano lipase PS, from Burkholderia cepacia (AM), lipase from Rhizopus niveus (RN), lipase acrylic resin from Candida antartica $(A R C)$ (recombinant, expressed in Aspergillus niger), Lipase $B$ Candida antartica immobilized on Immobead 150, recombinant from Aspergillus oryzae (CALB).

mulations can enhance protein stability by preventing/reducing protein-surface interactions. This has been observed for protein formulations with the addition of low molecular weight surfactants, such as polyoxyethylene sorbitan esters ${ }^{22}$.

\section{Effect of water content}

As previously mentioned (Results: screening of different NADESs for enzyme activity), the role of hydration water between the enzyme and the bulk ated with the rapidly hydrating sample. Possibly, the addition of a polymeric coating to protein for- 


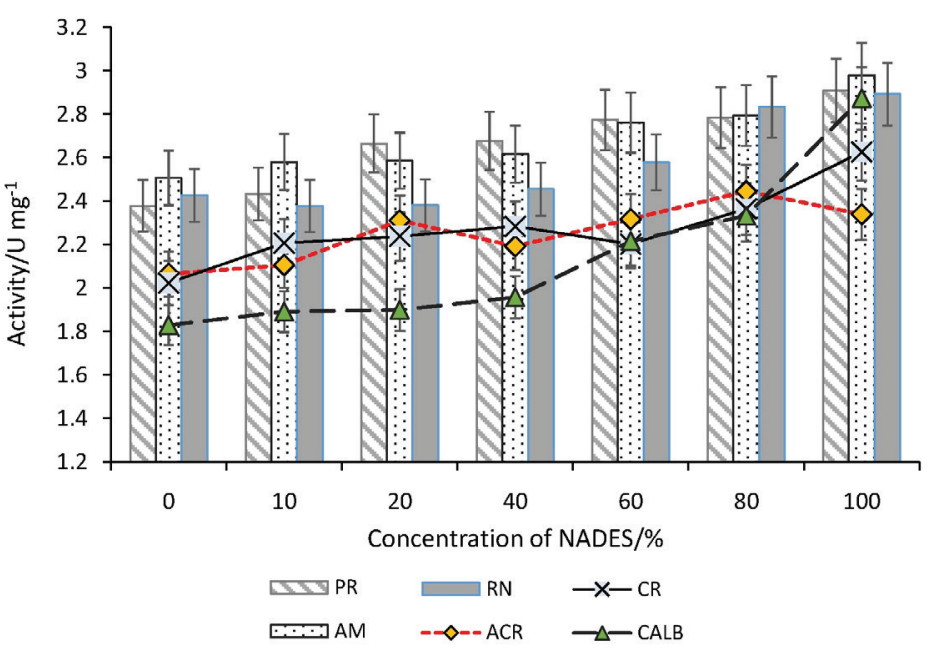

Fig. $5 \mathrm{a}$ - Activity of lipases at different concentrations of aqueous NADES3

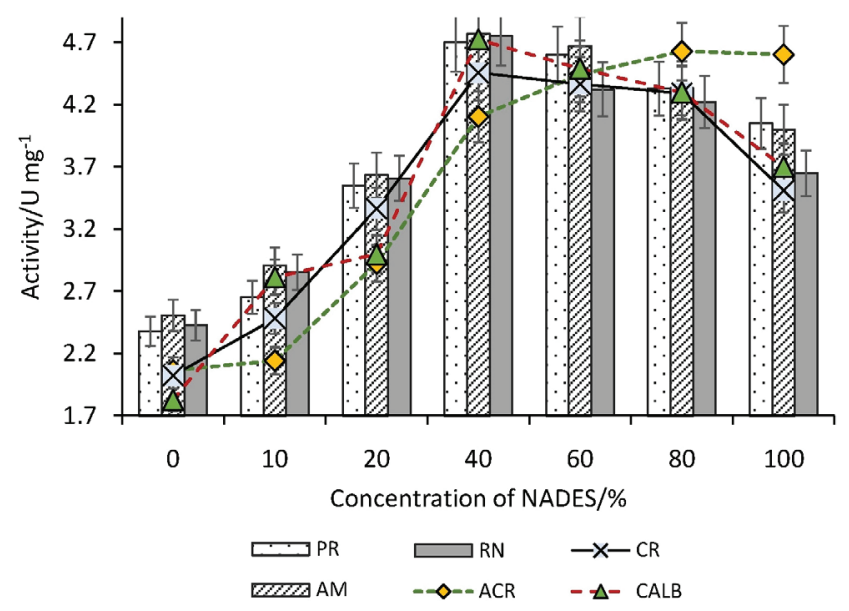

Fig. 5b - Activity of lipases at different concentrations of non-aqueous NADES3 (40\%). Lipase from porcine pancreas $(P R)$, lipase from Candida rugosa (CR), Amano lipase PS, from Burkholderia cepacia (AM), lipase from Rhizopus niveus (RN), lipase acrylic resin from Candida antartica (ARC) (recombinant, expressed in Aspergillus niger), Lipase B Candida antartica immobilized on Immobead 150, recombinant from Aspergillus oryzae (CALB).

solvent is the primary factor that differentiates molecular-level details in various media ${ }^{23}$. In the previous section, the reaction was conducted in the NADES without varying the water content for aqueous and NADES3a. Nevertheless, it was reported that water plays a crucial role in the three-dimensional organization of enzymes as well as maintaining the stability of protein ${ }^{24}$. Therefore, water was introduced to the reaction medium in different concentrations and the activity was observed. Figs. 5a and $5 \mathrm{~b}$ show the behavior of two different forms of NADES3. After an hour of incubation, the trend of the aqueous NADES3a can be seen from the activity monitoring. All lipases exhibited the same trend, as increasing the concentration of the NADES3a to water content increased the activity significantly until no water was added to the reaction media. ARC possessed a different flow as its activity was slightly reduced after increasing the concentration above $80 \%$.

In general, the observation supported the fact that a certain amount of water is required in the system for the enzyme to be hydrated, active or stable throughout the reaction time ${ }^{25}$. On the other hand, the non-aqueous NADES3 showed unalike behavior with lipases. All lipases used in this study displayed some level of improvement by increasing the water concentration in the media up to $60 \%(\mathrm{v} / \mathrm{v})$, except for ARC lipase, which showed higher activity at $20 \%$ $(\mathrm{v} / \mathrm{v})$ water in the system. It was also noticed that lipases started to show a slight reduction in their activities compared to the ones obtained at low concentrations of the non-aqueous NADES3. However, inhibition of the activities was not observed in any tested concentrations if compared with the initial activity (in buffered medium). ARC, though, was the most active at less water content as its activity reduced slightly at the least possible water content.

The results confirm that the hydrolytic activity of the lipase enzyme in NADES is influenced by the ratio of water in the solution. Regardless of the different effects of each solvent on different enzymes, a similar relationship between the amount of water added and the initial reaction rate was observed. It was reported that enzymes require more water in hydrophilic solvents than hydrophobic ones to obtain high catalytic activity ${ }^{26}$. The results of this study are, to some extent, consistent with earlier reports as it was conveyed that lipase-catalyzed reactions in DES are extremely difficult to occur without the presence of water ${ }^{27}$. Zhao et al. have so far reported expanded studies on DESs. They studied the selectivity of protease enzyme in glycerol-based DESs as solvents and found that both selectivity and activity of the enzyme is affected by water content ${ }^{15}$.

\section{Kinetic parameters of lipases in NADESs}

In order to confirm the lipase activity in the selected NADES, an additional investigation was conducted to assess the effect of NADES3 and NADE$\mathrm{S} 3 \mathrm{a}$ on the kinetic parameters compared with the phosphate buffer medium. The results obtained are presented in Table 3.

The Lineweaver-Burk plot for enzyme-catalyzed reaction following Michaelis-Menten model was used to evaluate the parameters from the experimental results. The kinetic studies showed that varying the media of the reaction directly influenced the Michaelis-Menten constant $\left(K_{\mathrm{m}}\right)$ and $V_{\max }$ values of the lipases involved in this study. The ac- 
Table 3 -Kinetic parameters of lipases in pNPP substrate solution using different reaction media

\begin{tabular}{|c|c|c|c|c|c|}
\hline Lipase & Reaction medium & $\begin{array}{c}K_{\mathrm{m}} \\
(\mathrm{mM})\end{array}$ & $\begin{array}{c}V_{\max } \\
\left(\mathrm{mM} \mathrm{min}^{-1}\right)\end{array}$ & $\begin{array}{c}k_{\text {cat }} \\
\min ^{-1}\end{array}$ & $\begin{array}{c}k_{\mathrm{cat}} / K_{\mathrm{m}} \\
\left(\mathrm{min} \mathrm{mM}^{-1}\right)\end{array}$ \\
\hline \multirow{3}{*}{ PR } & Phosphate buffer $8.0 \pm 0.2$ & $1.05 \pm 0.52$ & $3.38 \pm 1.21$ & $5.07 \pm 0.08$ & $4.83 \pm 1.05$ \\
\hline & Pure NADES3a & $0.78 \pm 0.09$ & $10.52 \pm 2.3$ & $15.75 \pm 1.02$ & $20.19 \pm 3.21$ \\
\hline & $40 \%$ NADES3 & $0.53 \pm 0.08$ & $13.35 \pm 3.5$ & $20.03 \pm 2.54$ & $37.79 \pm 4.22$ \\
\hline \multirow{3}{*}{$\mathrm{AM}$} & Phosphate buffer $8.0 \pm 0.2$ & $1.52 \pm 0.25$ & $4.91 \pm 1.02$ & $7.36 \pm 1.04$ & $4.84 \pm 0.08$ \\
\hline & Pure NADES3a & $0.68 \pm 0.07$ & $14.74 \pm 2.03$ & $22.11 \pm 2.28$ & $32.51 \pm 2.07$ \\
\hline & $40 \%$ NADES3 & $0.61 \pm 0.08$ & $16.28 \pm 2.24$ & $24.42 \pm 3.21$ & $40.03 \pm 3.15$ \\
\hline \multirow{3}{*}{$\mathrm{RN}$} & Phosphate buffer $8.0 \pm 0.2$ & $1.38 \pm 0.92$ & $3.37 \pm 1.32$ & $5.06 \pm 0.09$ & $3.67 \pm 0.09$ \\
\hline & Pure NADES3a & $0.66 \pm 0.08$ & $10.13 \pm 1.62$ & $15.20 \pm 1.52$ & $23.03 \pm 2.54$ \\
\hline & $40 \%$ NADES3 & $0.45 \pm 0.05$ & $12.05 \pm 2.05$ & $18.08 \pm 2.01$ & $40.18 \pm 3.25$ \\
\hline \multirow{3}{*}{ ACR } & Phosphate buffer $8.0 \pm 0.2$ & $1.25 \pm 0.25$ & $3.18 \pm 1.02$ & $4.77 \pm 1.23$ & $3.82 \pm 0.06$ \\
\hline & Pure NADES3a & $1.08 \pm 0.09$ & $3.05 \pm 0.08$ & $4.57 \pm 1.42$ & $4.23 \pm 0.05$ \\
\hline & $40 \%$ NADES3 & $0.49 \pm 0.05$ & $9.52 \pm 1.54$ & $13.88 \pm 1.67$ & $28.33 \pm 1.27$ \\
\hline \multirow{3}{*}{$\mathrm{CR}$} & Phosphate buffer $8.0 \pm 0.2$ & $1.55 \pm 0.35$ & $3.56 \pm 1.02$ & $5.34 \pm 0.08$ & $3.45 \pm 1.07$ \\
\hline & Pure NADES3a & $0.57 \pm 0.06$ & $10.69 \pm 2.24$ & $16.04 \pm 1.45$ & $28.14 \pm 2.07$ \\
\hline & $40 \%$ NADES3 & $0.45 \pm 0.07$ & $12.55 \pm 1.67$ & $18.83 \pm 2.07$ & $41.84 \pm 4.11$ \\
\hline \multirow{3}{*}{ CALB } & Phosphate buffer $8.0 \pm 0.2$ & $0.61 \pm 0.04$ & $2.03 \pm 0.91$ & $3.05 \pm 0.09$ & $5.00 \pm 1.04$ \\
\hline & Pure NADES3a & $0.52 \pm 0.03$ & $6.92 \pm 1.33$ & $10.38 \pm 2.05$ & $19.96 \pm 1.47$ \\
\hline & $40 \%$ NADES3 & $0.42 \pm 0.08$ & $15.52 \pm 1.85$ & $23.28 \pm 3.08$ & $55.43 \pm 3.26$ \\
\hline
\end{tabular}

tivation of the enzymatic activity could be clearly observed with a reduction in $K_{\mathrm{m}}$ value and an increase in $V_{\max }$. As can be seen in Table 3, upon using NADES3 and its aqueous form as co-solvent $(40 \%), K_{\mathrm{m}}$ slightly decreased in most cases with the lowest value observed with CALB. In comparison, when both NADES3a were used as the main solvent, the $K_{\mathrm{m}}$ values were slightly higher, with the highest obtained with ACR as it was observed to be more active in non-aqueous form.

The kinetics also confirmed the effect of both NADES3 and NADES3a on the velocity of all lipases used in the study. The largest $V_{\max }$ values were obtained in both AM and CALB with slight differences in the non-aqueous form as a co-solvent $(40 \%)$. However, the minimum value was attained in ACR in both aqueous and non-aqueous NADES. The results obtained confirmed the reports by ${ }^{28}$ that $\mathrm{ChCl}$ could enhance the enzymatic activity of yeast alcohol dehydrogenase by significantly increasing the affinity of the enzyme towards the substrate (reduces the value of $K_{\mathrm{m}}$ ). The highest turnover number $k_{\text {cat }}$ was obtained in $40 \%$ of NADES3 with PR, $\mathrm{AM}$ and CALB lipases. Furthermore, $k_{\mathrm{cat}} / K_{\mathrm{m}}$ denoted the catalytic efficiency for the CALB in $40 \%$ of NADES3 at $55.43 \pm 3.26 \mathrm{~min} \mathrm{mM}^{-1}$. Although CALB showed the highest catalytic efficiency, similar values were obtained for other lipases $\left(\sim 40\right.$ min $\left.\mathrm{mM}^{-1}\right)$, excluding ACR, which had the lowest efficiency. Lindberg et al. ${ }^{29}$ reported that turnover numbers were not affected by DESs as a co-solvent, whereas $K_{\mathrm{m}}$ increased when the concentrations increased. However, the results cannot be generalized to other enzymes or reactions as different lipases exhibited different activities in the NADESs used in the current study. Moreover, the nature and the structure of NADESs may be providing the advantage of higher stability in contrast with regular DESs.

\section{Storage of lipases in NADESs}

Fig. 6 shows the storage stability of lipase in aqueous solutions of NADES3 and NADES3a mixtures. The residual activity of lipases ranged between $80-97 \%$ of the initial activity after 72 hours in phosphate buffer at $4.0^{\circ} \mathrm{C}$. Lipase stored in NADES3 and NADES3a solutions showed higher relative activity than that stored in buffer, where it can be observed that the presence of NADES preserved the lipases for a long incubation period. Sugar-based NADES could maintain the lipase activity as no activity loss was detected after incubation.

\section{Discussion}

The trend of deactivation observed in this study could be attributed to the destabilization of the enzyme-substrate or reaction intermediate complexes at high concentrations of DESs, not due to protein 


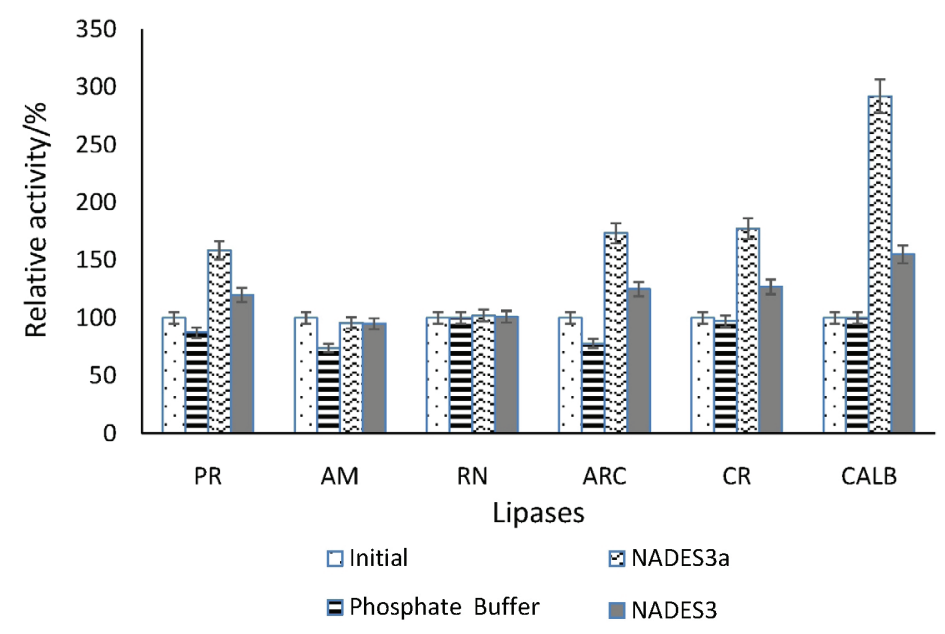

F ig. 6 - Relative activity of lipases after storage in NADES3, NADES3 $a$ and buffer compared to the initial activity for 72 hours at $4.0 \pm 2.0^{\circ} \mathrm{C}$. Lipase from porcine pancreas (PR), lipase from Candida rugosa (CR), Amano lipase PS, from Burkholderia cepacia (AM), lipase from Rhizopus niveus (RN), lipase acrylic resin from Candida antartica (ARC) (recombinant, expressed in Aspergillus niger), Lipase B Candida antartica immobilized on Immobead 150, recombinant from Aspergillus oryzae (CALB).

denaturation ${ }^{29}$. It was also reported by Gorke et al. ${ }^{11}$ that $25 \%$ increment for CALB was attained upon the addition of $10 \%(\mathrm{v} / \mathrm{v}) \mathrm{ChCl} / \mathrm{glycerol}$. When the conversion was $4.6 \%$ in the buffer, it increased to $92 \%$ in $25 \%(\mathrm{v} / \mathrm{v}) \mathrm{ChCl} /$ glycerol. The enhancement of the enzyme activity and stability in the NADESs can be explained by the presence of the hydrogen bonds between the salt and HBD, which causes a significant modification in the chemical nature of the resulting DES. This may have prompted expansion in hydrogen bond interactions, lessened the thermodynamic water activity in the reaction media, and therefore enhanced the enzyme activity ${ }^{15}$.

In this study, it was observed that the activity changed with $\mathrm{pH}$ changes. Any adjustment in $\mathrm{pH}$ can change the conditions of ionization of amino acids in protein as it has the zwitterion property. Thus, the charge properties of the substrate or state of a protein will be influenced either by preventing the substrate binding or inhibiting the catalysis ${ }^{30}$. It ought to be specified here that enzyme activity and the molar extinction coefficient of $p$ NP relied upon the $\mathrm{pH}$ as well as on the buffer utilized. It is well-observed that lipases preferred phosphate buffer $^{31}$. Moreover, the $\mathrm{pH}$ changes might also affect the NADES hydrogen bonding capacity due to low or high $\mathrm{pH}$ values that influence the presence of hydronium and hydroxyl ions in the solution.

Further investigation was executed to study the effect of reaction temperature on enzyme activity. The results obtained showed a typical enzyme activity trend with a variation in optimal reaction tem- peratures for each lipase in the range of 30 to $80^{\circ} \mathrm{C}$. Most of the lipases tested showed better stability at higher temperatures than their optimum when incubated with NADESs, which highlighted the role of NADESs in stability of proteins. Some excipients that preserve protein activity and maintain the stability are sugar-based. The NADESs used in this study were sugar-based and thus expected to stabilize the enzyme as a protein structure. Among a few, lactose, dextrose, mannitol and sucrose have been reported as binder agents, filler agents and microencapsulation agents for protecting bioactive compounds or active pharmaceutical compounds in many types of food and pharmaceutical products ${ }^{32}$.

The form of aqueous NADES3a contains water as one of its components $(\mathrm{ChCl} /$ sucrose/water; $4: 1: 4)$. Therefore, the water content available in the system might be sufficient, and only a low concentration was required for the enzyme to maintain its activity. On the other hand, non-aqueous NADES3 exhibited high viscosity, and thus was unable to dissolve the enzyme in the absence of water. However, it was observed that excess water had not led to enzyme deactivation, and that might be attributed to the nature of the DES used, which consisted of a sugar component. Some ILs and DESs have been stated to damage the water layer around the enzyme surface, or prevent access to the active site, and accordingly, reduce the activity and stability of the enzyme ${ }^{15}$.

The increment in activity of the lipases observed in NADES3 with water addition could also be attributed to the viscosity reduction as water can decrease the viscosity and change the activity and selectivity of the enzyme. On the other hand, water is the simplest hydrogen-bond donor. Thus, its content should be as low as possible to avoid any possible change in the initial eutectic composition and prevent competitive hydrolysis reaction'. The results obtained are in consistency with reports conveying that water addition to DESs strongly improves both enzyme activity and reaction yield ${ }^{33}$. In addition, studies have revealed that the shallow lipase activity/stability in the neat or weakly hydrated DES for such complex experiments could be explained by both the strong association of the substrate in the DES matrix and the shallow thermodynamic water activity $\left(a_{w}<0.02\right)$. Indeed, significant enhancement in initial lipase activity $(67$ times faster in $\mathrm{ChCl} / \mathrm{U}$ ) was observed when $a_{\mathrm{w}}$ increased up to 0.15 . Also, water in DES significantly preserved the enzyme catalytic activity over time ${ }^{24}$.

\section{Conclusion}

Among the NADESs tested in this study, NADES prepared from $\mathrm{ChCl}$ and sucrose was selected as the most promising solvent as it has proven to be 
effective in the activation of most of the lipases used or stabilizes them compared to other NADESs applied. The structure of NADES seems to have protective properties rather than only enzyme activation. The notable improvement of the activity in NADES may be attributed to the hydrogen bonding ability between the salt and the HBD, which enhances the enzyme affinity. It may also be in relation to the non-toxic property of the NADES. Lipases mostly maintained the initial activity in NADES even at $70{ }^{\circ} \mathrm{C}-80{ }^{\circ} \mathrm{C}$ for a period of $1 \mathrm{~h}$. In contrast, the activity in phosphate buffer started to drop at $60{ }^{\circ} \mathrm{C}$. The comparison between aqueous and non-aqueous NADES showed that lipases preferred the non-aqueous NADES with $40 \%(\mathrm{v} / \mathrm{v})$ of NADES to water concentration. This may be associated with the high viscosity of the non-aqueous NADES and the low solubility of lipases in the absence of water. Remarkably, NADESs are gaining attention in research due to their catalytic activity, and also their sustainability and biodegradability. This approach of using NADES as green solvents might be a good choice for eco-friendly production of biodiesel and the extraction of bioactive compounds.

\section{ACKNOWLEDGMENT}

The authors would like to express their gratitude to Bantuan Kecil Penyelidikan (BKP) grant no. BK0332017 and UMRG grant RP037B-15AET for their support to this research.

\section{CONFLICT OF INTEREST}

The authors declare that they have no conflict of interest.

\section{References}

1. Smith, E. L., Abbott, A. P., Ryder, K. S., Deep eutectic solvents (DESs) and their applications, Chem. Rev. 114 (2014) 11060 doi: https://doi.org/10.1021/cr300162p

2. Dai, Y., van Spronsen, J., Witkamp, G.-J., Verpoorte, R., Choi, Y. H., Natural deep eutectic solvents as new potential media for green technology, Anal. Chim. Acta 766 (Supplement C) (2013) 61 . doi: https://doi.org/10.1016/j.aca.2012.12.019

3. Kudlak, B., Owczarek, K., Namieśnik, J., Selected issues related to the toxicity of ionic liquids and deep eutectic solvents-A review, Environ. Sci. Pollut. Res. 22 (2015) 11975 . doi: https://doi.org/10.1007/s11356-015-4794-y

4. Panic, M., Radosevic, K., Bubalo, M. C., Vidović, C., Redovnikovic Radojcic, I., Jokic, S., Green solvents for green technologies, J. Biotechnol. 256 (2017) (Supplement) S11-S12.

doi: https://doi.org/10.1016/j.jbiotec.2017.06.040
5. Paiva, A., Craveiro, R., Aroso, I., Martins, M., Reis, R. L., Duarte, A. R. C., Natural deep eutectic solvents - solvents for the 21st century, ACS Sustain. Chem. Eng. 2 (2015) 1063 .

doi: https://doi.org/10.1021/sc500096j

6. Kumar, A. K., Parikh, B. S., Pravakar, M., Natural deep eutectic solvent mediated pretreatment of rice straw: Bioanalytical characterization of lignin extract and enzymatic hydrolysis of pretreated biomass residue, Environ. Sci. Pollut. Res. 23 (2016) 9265. doi: https://doi.org/10.1007/s11356-015-4780-4

7. Espino, M., de los Ángeles Fernández, M., Gomez, F. J. V., Silva, M. F., Natural designer solvents for greening analytical chemistry, TrAC - Trends Anal. Chem. 76 (2016) 126. doi: https://doi.org/10.1016/j.trac.2015.11.006

8. Zhao, H., Zhang, C., Crittle, T. D., Choline-based deep eutectic solvents for enzymatic preparation of biodiesel from soybean oil, J. Mol. Catal. B Enzym. 85-86 (2013) 243. doi: https://doi.org/10.1016/j.molcatb.2012.09.003

9. Durand, E., Lecomte, J., Baréa, B., Piombo, G., Dubreucq, $E$., Villeneuve, $P$., Evaluation of deep eutectic solvents as new media for Candida antarctica B catalyzed reactions, Process Biochem. 47 (2012) 2081. doi: https://doi.org/10.1016/j.procbio.2012.07.027

10. Zhao, H., Baker, G., Holmes, S., New eutectic ionic liquids for lipase activation and enzymatic preparation of biodiesel, Org. Biomol. Chem. 9 (2011) 1908. doi: https://doi.org/10.1039/c0ob01011a

11. Gorke, J. T., Srienc, F., Kazlauskas, R. J., Hydrolase-catalyzed biotransformations in deep eutectic solvents, Chem. Commun. 10 (2008) 1235. doi: https://doi.org/10.1039/B716317G

12. Hayyan, A., Mjalli, F. S., Alnashef, I. M., Al-Wahaibi, T., Al-Wahaibi, Y. M., Hashim, M. A., Fruit sugar-based deep eutectic solvents and their physical properties, Thermochim. Acta 541 (2012) 70. doi: https://doi.org/10.1016/j.tca.2012.04.030

13. Nomanbhay, S. M., Hussain, R., Palanisamy, K., Microwave-assisted alkaline pretreatment and microwave assisted enzymatic saccharification of oil palm empty fruit bunch fiber for enhanced fermentable sugar yield, J. Sustain. Bioenergy Syst. 3 (2013) 7. doi: https://doi.org/10.4236/jsbs.2013.31002

14. Kim, S. H., Park, S., Yu, H., Kim, J. H., Kim, H. J., Yang, Y. H., Kim, Y. H., Kim, K. J., Kan, E., Lee, S. H., Effect of deep eutectic solvent mixtures on lipase activity and stability, J. Mol. Catal. B Enzym. 128 (2016) 65. doi: https://doi.org/10.1016/j.molcatb.2016.03.012

15. Zhao, H., Baker, G. A., Holmes, S., Protease activation in glycerol-based deep eutectic solvents, J. Mol. Catal. B Enzym. 72 (2011) 163. doi: https://doi.org/10.1016/j.molcatb.2011.05.015

16. Zisis, T., Freddolino, P. L., Turunen, P., van Teeseling, M. C. F., Rowan, A. E., Blank, K. G., Interfacial activation of Candida antarctica lipase B: Combined evidence from experiment and simulation, Biochemistry 54 (2015) 5969 doi: https://doi.org/10.1021/acs.biochem.5b00586

17. Erwann, D., Jérôme, L., Pierre, V., Are emerging deep eutectic solvents (DES) relevant for lipase-catalyzed lipophilizations? Oilseeds fats Crop Lipids. 22 (2015) 1. doi: https://doi.org/10.1051/ocl/2015026

18. Merza, F., Fawzy, A., AlNashef, I., Al-Zuhair, S., Taher, H., Effectiveness of using deep eutectic solvents as an alternative to conventional solvents in enzymatic biodiesel production from waste oils, Energy Reports 4 (2018) 77. doi: https://doi.org/10.1016/j.egyr.2018.01.005 
19. Juneidi, I., Hayyan, M., Hashim, M. A., Hayyan, A., Pure and aqueous deep eutectic solvents for a lipase-catalysed hydrolysis reaction, Biochem. Eng. J. 117 (2017) 129. doi: https://doi.org/10.1016/j.bej.2016.10.003

20. Naushad, M., ALOthman, Z. A., Khan, A. B., Ali, M., Effect of ionic liquid on activity, stability, and structure of enzymes: A review, Int. J. Biol. Macromol. 51 (2012) 555. doi: https://doi.org/10.1016/j.ijbiomac.2012.06.020

21. Sanghamitra, N. J. M., Ueno, T., Stability and activity of enzymes in ionic liquids BT, in Mohammad, A. and Inamuddin, D. (Eds.), Green Solvents II: Properties and Applications of Ionic Liquids, Springer Netherlands, Dordrecht, 2012, pp 235-273. doi: https://doi.org/10.1007/978-94-007-2891-2_10

22. Elversson, J., Millqvist-Fureby, A., In situ coating-An approach for particle modification and encapsulation of proteins during spray-drying, Int. J. Pharm. 323 (2006) 52. doi: https://doi.org/10.1016/j.ijpharm.2006.05.066

23. Fogarty, A. C., Laage, D., Water dynamics in protein hydration shells: the molecular origins of the dynamical perturbation, J. Phys. Chem. B. 118 (2014) 7715. doi: https://doi.org/10.1021/jp409805p

24. Durand, E., Lecomte, J., Villeneuve, P., Deep eutectic solvents: Synthesis, application, and focus on lipase-catalyzed reactions, Eur. J. Lipid Sci. Technol. 115 (2013) 379. doi: https://doi.org/10.1002/ejlt.201200416

25. Phisut, N., Spray drying technique of fruit juice powder: some factors influencing the properties of product, Int. Food Res. J. 19 (2012) 1297.

26. Yang, L., Dordick, J. S., Garde, S., Hydration of enzyme in nonaqueous media is consistent with solvent dependence of its activity, Biophys. J. 87 (2017) 812.

doi: https://doi.org/10.1529/biophysj.104.041269

27. Aouf, C., Durand, E., Lecomte, J., Figueroa-Espinoza, M. C., Dubreucq, E., Fulcrand, H., Villeneuve, P., The use of lipases as biocatalysts for the epoxidation of fatty acids and phenolic compounds, Green Chem. 16 (2014) 17406. doi: https://doi.org/10.1039/c3gc42143k

28. Weibels, S., Syguda, A., Herrmann, C., Weingartner, H., Steering the enzymatic activity of proteins by ionic liquids. A case study of the enzyme kinetics of yeast alcohol dehydrogenase, Phys. Chem. Chem. Phys. 14 (2012) 4635. doi: https://doi.org/10.1039/C2CP24041F

29. Lindberg, D., de la Fuente Revenga, M., Widersten, M., Deep eutectic solvents (DESs) are viable cosolvents for enzyme-catalyzed epoxide hydrolysis, J. Biotechnol. 147 (2010) 169. doi: https://doi.org/10.1016/j.jbiotec.2010.04.011

30. del Monte, F., Carriazo, D., Serrano, M. C., Gutiérrez, M. C., Ferrer, M. L., Deep eutectic solvents in polymerizations: A greener alternative to conventional syntheses, Chem. Sus. Chem. 7 (2014) 999. doi: https://doi.org/10.1002/cssc.201300864

31. Yang, Z., Zhang, K. P., Huang, Y., Wang, Z., Both hydrolytic and transesterification activities of Penicillium expansum lipase are significantly enhanced in ionic liquid [BMIm] [PF ] J. Mol. Catal. B Enzym. 63 (2010) 23. doi: https://doi.org/10.1016/j.molcatb.2009.11.014

32. Amid, A., Arshad, Z. I. M., Othman, M. E. F., Case Study: Recombinant bromelain downstream processing BT, in Amid, A. (Ed.), Recombinant Enzymes-From Basic Science to Commercialization, Springer International Publishing, Switzerland, 2015, pp 175-185. doi: https://doi.org/10.1007/978-3-319-12397-4_12

33. Cvjetko-Bubalo, M., Jurinjak-Tušek, A., Vinković, M., Radošević, K., Gaurina-Srček, V., Radojčić-Redovniković, I., Cholinium-based deep eutectic solvents and ionic liquids for lipase-catalyzed synthesis of butyl acetate, J. Mol. Catal. B Enzym. 122 (2015) 188. doi: https://doi.org/10.1016/j.molcatb.2015.09.005 